\title{
Probiotics, gut microbiota, and their influence on host health and disease
}

\author{
Borja Sánchez ${ }^{1}$, Susana Delgado ${ }^{1}$, Aitor Blanco-Míguez ${ }^{2}$, Anália Lourenço ${ }^{2,3}$, \\ Miguel Gueimonde ${ }^{1}$ and Abelardo Margolles ${ }^{1}$ \\ ${ }^{1}$ Department of Microbiology and Biochemistry of Dairy Products, Instituto de Productos Lácteos de Asturias \\ (IPLA), Consejo Superior de Investigaciones Científicas (CSIC), Villaviciosa, Asturias, Spain \\ ${ }^{2}$ ESEI - Department of Computer Science, University of Vigo, Edificio Politécnico, Campus Universitario As Lagoas \\ s/n 32004, Ourense, Spain \\ ${ }^{3}$ CEB - Centre of Biological Engineering, University of Minho, Campus de Gualtar, Braga, Portugal
}

The gastrointestinal tract of mammals hosts a high and diverse number of different microorganisms, known as intestinal microbiota. Many probiotics were originally isolated from the gastrointestinal tract, and they were defined by the Food and Agriculture Organization of the United Nations (FAO)/WHO as "live microorganisms which when administered in adequate amounts confer a health benefit on the host." Probiotics exert their beneficial effects on the host through four main mechanisms: interference with potential pathogens, improvement of barrier function, immunomodulation and production of neurotransmitters, and their host targets vary from the resident microbiota to cellular components of the gut-brain axis. However, in spite of the wide array of beneficial mechanisms deployed by probiotic bacteria, relatively few effects have been supported by clinical data. In this regard, different probiotic strains have been effective in antibiotic-associated diarrhea or inflammatory bowel disease for instance. The aim of this review was to compile the molecular mechanisms underlying the beneficial effects of probiotics, mainly through their interaction with the intestinal microbiota and with the intestinal mucosa. The specific benefits discussed in this paper include among others those elicited directly through dietary modulation of the human gut microbiota.

\section{Keywords:}

Gut microbiota / Health effects / Molecular mechanisms bioinformatics / Probiotics

\section{Introduction}

The gastrointestinal tract (GIT) of mammals hosts a high and diverse number of different microorganisms, known as intestinal microbiota. Bacteria, Archea, fungi protozoa, and viruses cohabit and interact mutually and with the host, notably with epithelial and immune cells. Gut microbe populations have achieved a more or less defined configuration dur-

Correspondence: Borja Sánchez

E-mail: borja.sanchez@csic.es

\begin{abstract}
Abbreviations: AAD, antibiotic-associated diarrhea; CDAD, Clostridium difficile-associated diarrhea; ESPGHAN, European Society for Paediatric Gastroenterology Hepatology and Nutrition; FAO, Food and Agriculture Organization of the United Nations; GALT, gut-associated lymphoid tissue; GIT, gastrointestinal tract; IBD, inflammatory bowel disease; IBS, irritable bowel syndrome; NGS, next-generation sequencing; UC, ulcerative colitis; WAO, World Allergy Organization; WGO, World Gastroenterology Organization; WHO, World Health Organization
\end{abstract}

Received: March 16, 2016

Revised: June 29, 2016

Accepted: July 14, 2016 ing human evolution, which has facilitated the establishment of mutualist relationships with the host [1]. During the last 10 years, development of next generation DNA sequencing platforms has allowed a deep understanding of the composition of the microbial populations inhabiting not only the gut, but other locations in the body, such as the upper airways of the respiratory tract, the skin, the mouth, or the vagina. Given the metabolic potential encoded into the genes harbored by our intestinal microbiota, at least 50 -fold more unique genes than our own human genes, many researchers are beginning to consider our intestinal microbiota as another organ of the human body. Indeed, our gut microbes are implicated in the normal physiology of our GIT, and alterations in the relative amounts of their populations can disrupt the beneficial interactions between microbiota and host, having a direct effect on human health [2].

Different functions are being attributed to the intestinal microbiota in light of the research conducted mainly in the last 10 years, among which (i) maintenance of the epithelial 
barrier, (ii) inhibition of pathogen adhesion to intestinal surfaces, (iii) modulation and proper maturation of the immune system, (iv) degradation of otherwise nondigestible carbon sources such as plant polysaccharides, and (v) production of different metabolites such as vitamins and SCFAs, are noteworthy. Perhaps the best indication of the beneficial influence of the intestinal microbiota over the host has been obtained from germ-free animals, in which the absence of microorganisms colonizing their digestive tube ends in an immature immune system and an aberrant GIT [3]. For instance, the number of Peyer's patches is lower, as well as the number of colonic crypts, intraepithelial lymphocytes, Goblet cells, and therefore a thinner mucus layer; contrary to this the caecum is notably increased in volume with respect to standard animals [4]. However, these models display several limitations, notably the absence of a mature immune system, which might influence the functionality of the gut microbiota. In germ-free animals, withdrawal of sterile conditions or fecal transplantation are ways of inducing immune system maturation [5].

During the last few years, some multidisciplinary initiatives have notably contributed to our understanding of the gut microbiota, among which large collaborative projects, such as the MetaHit project (http://www.metahit.eu/), the Human Microbiome Project (http://hmpdacc.org/), or the MyNewGut project (http://www.mynewgut.eu/). Research conducted in the framework of these projects has generated enough evidence to provide a qualitative change in the importance of our gut microbes, mainly by the characterization of the microbial communities associated to different human diseases and the definition of environmental factors affecting their dynamics, as well as by the creation of a genetic catalogue compiling all reference genes of microbial origin [6-9] (http://gigadb.org/dataset/view/id/100064).

In this regard, there is a great deal of controversy about what is the proper composition of the human gut microbiota, mainly because there is high interindividual variability in terms of microbial abundances and because the relative proportions of our intestinal microbes are highly influenced by environmental factors, such as dietary patterns and other habits [10]. In addition, intestinal microbiota is composed of autochthonous members colonizing the gut mucosa, and transient microbiota which is part of the ingested foods. It has been estimated that the gut microbiota in each individual comprises more than 100 different species. Overall, about 1500 different species have been identified so far as being part of the human gut microbiota. A total number of $10^{13}-10^{14}$ total microbial cells constitute the intestinal microbiota, and it has been largely assumed that it represents ten times more cells than our own eukaryotic cells. However, this ratio is considered nowadays exaggerated, as for instance if we take into account the number of red cells, which has no nucleus, the 10:1 ratio looks to be more like 1:1 [11]. On the contrary, defecation may flip this proportion to 10:1 again, making this topic hot and controversial as a part of readers may consider a red cell to be an eukaryotic cell, while the other part will consider them differentiated cells that lose their nu- cleus to maximize their capacity to transport oxygen. The large intestine is by far the body location with the highest microbial concentration, about $10^{11}-10^{12}$ cells/g of intestinal content, representing $50-60 \%$ of the dry weight of feces [12]. Bacteria dominate the gut microbiota, being represented principally by the phyla Firmicutes and Bacteroidetes, and by secondary phyla such as Actinobacteria, Proteobacteria, Synergistetes, Fusobacteria, and Verrucomicrobia [13]. Fungi and Archea represent up to $1 \%$ of the species of the human gut microbiota [14]. Among the main representative genera of these phyla, Bacteroides sp., Faecalibacterium sp., Blautia sp., Prevotella sp., Clostridium sp., Ruminococcus sp., and Bifidobacterium sp. (in breast-fed infants) are noteworthy due to their high relative abundance, and indeed each and every one of us harbors several grams of one or more of these bacterial genera $[9,15]$.

Recently, it has been proposed that all the interindividual variability of the intestinal microbiota could be classified into three groups, the so called enterotypes, which are defined as a network of coabundant microbial populations dominated by the prominent presence of one of these three genera: $R u$ minococcus, Bacteroides, and Prevotella [16]. This classification has been a source of controversy, as some authors consider enterotypes a very simplistic model, reducing the whole intestinal microbiota complexity into three groups [17]. For instance, some authors have found just two of these enterotypes [18] as two continuous clusters of microbiota configurations separated by a gradient of species with variable abundances [19].

Therefore, considering the complexity of intestinal microbiota, it is unlikely that the diversity could be fitted into simple classification systems such as enterotypes. Categorizing the intestinal microbiota into enterotypes or other groups, with strong correlations with dietary patterns, could be extremely helpful in personalizing the treatment of diseases progressing with microbial dysbiosis [20]. This will require development of mathematical models able to condense the whole complexity, and therefore more experimental data are needed [21].

\section{The probiotic concept-Evolution}

Today the characterization of intestinal microbiota composition and activity is a hot topic for scientists working at the intersection of the fields of human Microbiology, Immunology and Health, and it has a direct connection with research on probiotic bacteria. Since ancient times fermented products containing living microorganisms have been used to restore or maintain health. This was already acknowledged in a version of the Old Testament that attributed the longevity of Abraham to the consumption of sour milk [22]. More recently, at the beginning of the last century, the Nobel laureate Russian immunologist Elie Metchnikoff introduced the idea of consuming beneficial live microorganisms to improve health. Metchnikoff proposed that eating fermented milks 
containing lactobacilli might displace putrefactive and pathogenic intestinal organisms and increase longevity [23]. This could be considered as the first reference to our current understanding of the probiotic concept.

Although the probiotic concept seems to be old, the term "probiotic" was not defined until recently. The first use of the term is attributed to Lilly and Stillwell who, in a paper published in Science in 1965, defined probiotics as "growthpromoting factors produced by microorganisms" [24]. In opposition to antibiotics, Lilly and Stillwell used this term to refer to substances produced by one microorganism stimulating the growth of others.

The first definition of probiotics, within the conceptual framework initiated by Metchnikoff, was that of Parker [25], who defined probiotics as "organisms and substances which contribute to intestinal microbial balance". A decade later, following the same concept but narrowing it to live microorganisms, Fuller proposed the definition "live microbial feed supplements which beneficially affect the host animal by improving its intestinal microbial balance" [26]. A few years later, to underline the microbial nature of probiotics and to explicitly cover any body habitat also in humans, a new definition was proposed as "viable mono- or mixed culture of microorganisms which, applied to animals or man, beneficially affects the host by improving the properties of the indigenous microflora" [27]. All these definitions have in common a focus on the effect upon the intestinal microbiota. However, there were an increasing number of scientific studies showing effects of probiotics not related to the intestinal microbiota, such as those demonstrating immune modulating capabilities. This led to the simultaneous proposition of two new definitions of probiotics as "live microbial culture or cultured dairy product which beneficially influences the health and nutrition of the host" [28] and as "living microorganisms which upon ingestion in certain numbers, exert health benefits beyond inherent basic nutrition" [29].

In this context the International Life Sciences Institute Europe (ILSI) tried to find a consensus definition and in 1998 a working group of experts proposed the definition "viable microbial food supplement which beneficially influences the health of the host" [30]. The same working group defined probiotic foods as functional if they "have been satisfactorily demonstrated to beneficially affect one or more target functions in the body beyond adequate nutritional effects, in a way that is relevant to either an improved state of health and well-being and/or reduction in the risk of diseases" [31]. Definitions covering dead bacteria and bacterial components were also proposed, such as "microbial cell preparations or components of microbial cells that have a beneficial effect on the health and well-being of the host" [32]. This definition did not require viable bacteria and was based on studies showing that non-viable bacteria and bacterial components (e.g. DNA) also produce some beneficial effects [33,34].

Attempts to find a consensus definition finally came to an end when in 2001 a panel of experts convened by the Food and Agriculture Organization of the United Nations (FAO)/ World Health Organization (WHO) proposed to define probiotics as "live microorganisms which when administered in ad- equate amounts confer a health benefit on the host" [35]. This definition was compiled together with recommendations regarding probiotic research in a guideline issued in 2012 by the FAO/WHO working group, and seems to have received enough quorum and consensus. This definition has been widely accepted since then and has been subjected only to a minor grammatical modification "live microorganisms that, when administered in adequate amounts, confer a health benefit on the host" [36]. According to this definition, which covers well our current probiotic concept, being a probiotic is a strain-specific characteristic. Thus, the properties of each probiotic strain should be well defined and cannot be extrapolated to other strains. It is necessary to scientifically demonstrate the efficacy of the strain in conferring a health benefit on the host, but this effect does not have to be linked to any specific mechanism of action.

The concept is not restricted to foods, allowing for other delivery options such as pharmaceutical preparations and other scenarios such as extra intestinal locations such as respiratory tract, skin, blood, genitourinary tracts etc. It does not involve an oral application either, allowing other delivery routes and topic application. Moreover, according to the current definition a probiotic must be alive, viable bacteria, and it is not applicable to dead bacterial cells or cell components. This FAO/WHO definition has been adopted by several organizations such as the International Scientific Association for Probiotics and Prebiotics (ISAPP), Codex, Institute of Food Technologists (IFT), World Gastroenterology Organization (WGO), or the European Food Safety Authority (EFSA) [36].

\section{Evidence-based probiotic effects on intestinal diseases}

A large number of disease states and intestinal disorders have been related to an unbalanced GIT microbiota. Scientific evidence supports the important roles that probiotics can play in the digestive system, having significant effects in alleviating the symptoms of several diseases [37]. This section reports on scientific evidence of benefit from probiotics for specific intestinal diseases, the main target of probiotic intervention. It summarizes the main illnesses of the GIT for which restoration of the microbial balance by the use of probiotics has evidenced beneficial health effects in the prevention or treatment of disease.

(i) Infectious diarrhea.

Of the various microorganisms that cause infectious diarrhea, rotavirus is the most common case in infants. Acute diarrhea is also frequent among travelers, in whom enterotoxigenic Escherichia coli is particularly common. Other important diarrheogenic bacterial pathogens are members of the genera Salmonella, Campylobacter, and Shigella. The rationale for using probiotics in infectious diarrhea is that they act against enteric pathogens by competing for available nutrients and binding sites, 
making the gut contents acid, producing a variety of chemicals, and increasing specific and nonspecific immune responses. Probiotics have been tried in a large number of clinical studies as a supplement to rehydration therapy in the treatment of infectious diarrhea. The results have been positive and remarkably consistent in shortening the duration and reducing stool frequency [38]. Although increasing numbers of travelers are also considering the use of probiotics as a preventive, there are fewer studies on adults and they are less conclusive $[37,39]$. However, the European Society for Paediatric Gastroenterology Hepatology and Nutrition (ESPGHAN) recommends consideration of probiotic administration in addition to rehydration as therapy for acute gastroenteritis in children: Lactobacillus rhamnosus GG, Saccharomyces boulardii and Lactobacillus reuteri DSM 17938, although the latter has very low quality of evidence according to the ESPGHAN report [40]. The World Allergy Organization (WAO) recently published their Guidelines for Allergic Disease Prevention (GLAD-P) based on the use of probiotics [41]. The WAO published recommendations about the use of probiotics in the prevention on allergy, based on scientific evidence and from the results of human trials. These guidelines conclude that currently there is no evidence supporting probiotic supplementation for reducing the risk of allergy incidence in children. However, there is likely net benefit using probiotics for eczema prevention, which requires more clinical trials to increase sample size. On the contrary, WAO suggests the use of probiotics in pregnant women, in women who breastfeed their infants and in the own infants, in all those cases where a family history of allergy (eczema) is identified as factor risk for children [41].

(ii) Antibiotic-associated diarrhea (AAD).

Antimicrobial therapy disturbs the endogenous GIT microbiota, frequently resulting in diarrhea. In the majority of the cases, this diarrhea is related to the overgrowth of $C$. difficile causing what is called $C$. difficile-associated diarrhea (CDAD). The increasing number of clinical studies in recent years provided data for meta-analyses testing the efficacy of probiotics (mainly the strain Lactobacillus GG and the yeast species $S$. boulardii) in the protection and relapse of CDAD. Recent systematic reviews with meta-analysis showed the effectivity of these probiotics in preventing AAD in both children and adults $[42,43]$. The ESPGHAN Probiotic Group does not recommend the use of probiotics for the treatment of AAD, as not enough randomized controlled trials are available. However, administration of probiotics for preventing AAD is recommended if some risk factors such as duration of the antibiotic treatment or comorbidities are present. In these cases, administration of L. rhamnosus GG and S. boulardii is strongly recommended by the ESPGHAN [44]. In the case of CDAD, and although the ESPGHAN consider administration of S. boulardii, fecal microbiota transplantation appears as a powerful treatment for the definitive eradication of this pathogen from the human gut [45].

(iii) Inflammatory bowel disease (IBD).

IBD includes Crohn's disease, ulcerative colitis (UC), and pouchitis, which are characterized by deregulation of the immune system leading to inflammation of the GIT. A disturbance in the GIT microbiota, or an inadequate host response to it, has been shown to play a critical role in the pathogenesis of IBD. Probiotics can influence both the GIT microbiota and the immune system and many experts in the field of IBD are using probiotics to treat IBD patients. The greatest evidence for efficacy of probiotics in these pathologies is in its use (in particular the formulation of multiple strains VSL3) for the prevention of pouchitis [46]. Trials examining the use of probiotics with Crohn's disease have been rather disappointing, while the evidence of benefit from probiotics in UC is quite promising [47].

(iv) Irritable bowel syndrome (IBS).

IBS is characterized by abdominal discomfort or pain and altered bowel function. Although the causes of IBS are not completely understood, growing evidence suggests a potential role of intestinal microbiota in its pathophysiology and symptom generation. For that, different probiotic intervention studies have been performed in last few years and although the results suggest that there is a benefit of probiotic use in the relief of IBS symptoms [37,48], welldesigned, placebo-controlled studies are recommended since each probiotic strain has unique features and IBS patients are heterogeneous [49].

(v) Helicobacter pylori infection.

Helicobacter pylori, a highly prevalent pathogen, is a major cause of chronic gastritis and peptic ulcers and a risk factor for gastric malignancies. Interventional studies using probiotics alone have not resulted in eradication of $H$. $p y$ lori. However, several trials have found that if probiotics are used in conjunction with the standard therapy, the rate of eradication is improved. Furthermore, probiotics (such as S. boulardii, L. reuteri, and L. rhamnosus GG) can help by preventing gastrointestinal antibiotic associated side effects [50,51].

(vi) Lactose intolerance.

A large part of the world population shows low levels of the enzyme $\beta$-galactosidase (lactase) in the mucosa of the small intestine. These individuals suffer intolerance symptoms when milk (lactose) is present in their diet. There is good scientific evidence for the alleviation of lactose intolerance symptoms by the consumption of yogurt and fermented milk products containing conventional lactic acid bacteria as starter cultures and probiotic bacteria [52]. These are thought to confer beneficial effects by providing microbial $\beta$-galactosidase activity that improves tolerance and digestibility of lactose. 


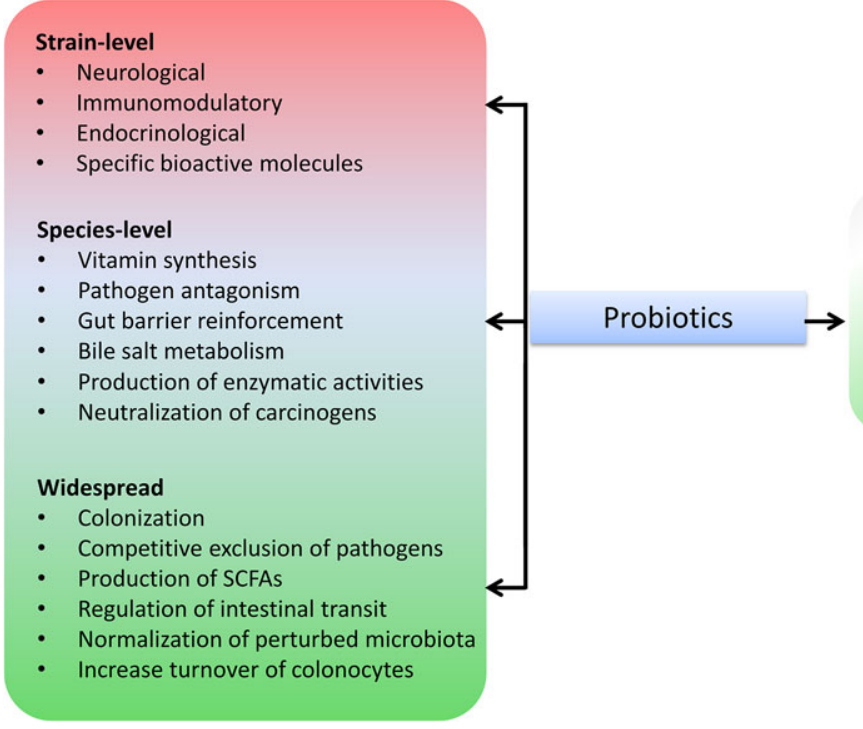

Evidence-based effects

- Infectious Diarrhea

- Antibiotic-Associated Diarrhea

Inflammatory Bowel Disease

Irritable Bowel Syndrome

- Helicobacter pylori infection

Lactose Intolerance

\section{Host-probiotic interaction-Mechanisms involved}

The probiotic microorganisms most commonly used in human nutrition are certain strains belonging to the genus Lactobacillus, Bifidobacterium, Enterococcus as well as strains from the genus Propionibacterium and certain yeasts such as $S$. boulardii [53]. During recent years, a high number of studies using animal models and clinical interventions have explored the potential benefits of probiotics on human health, mainly in the prevention and treatment of several diseases [54]. Probiotics exert their benefits through four different mechanisms of action: (i) interference with pathogenic bacteria by competing with nutrients and adhesion sites, (ii) improvement of the barrier function of the epithelial lining, (iii) immunomodulation, and (iv) influence on other organs of the body through the immune system and neurotransmitter production (such as $\gamma$-aminobutyric acid (GABA) or serotonin) (Fig. 1) [36]. For instance, probiotics should have a high adherence ability to the intestinal epithelium, limiting the access of enteropathogens to target molecules present on the host cell surfaces that are used for initiating the process of invasion. In addition, probiotics produce antimicrobial substances such as bacteriocins and SCFAs, inhibiting the growth of potential pathogens. Intestinal microbiota and some emerging probiotics have genes coding for proteins responsible for biochemical pathways not present in the human host, such as degradation of complex carbon source or vitamin and other essential compound production. For instance some strains produce butyrate as a result of their fermentative metabolism, which is a key molecule in the maintenance of the gut homeostasis notably at the level of epithelial cells.

Probiotics interact with the human host at three different levels of the lower part of the human GIT: mucus layer, epithelial layer, and gut-associated lymphoid tissue (GALT) (Fig. 2). The mucus layer is in turn subdivided into two sec- tions, an outer sublayer in which the mucin molecules form a quite relaxed gel, which is characterized by the presence of relatively high numbers of bacteria, as well as by the presence of antimicrobial peptides and secreted immunoglobulin A, aimed at limiting the amount of commensal bacteria colonizing this location. Contrary to this, there is an inner, dense, and compact mucin sublayer immediately adjacent to the epithelial cells, restricting access to the bacteria, and where few, or no, microorganisms are observed $[55,56]$.

The second level of interaction of probiotics with the human host is epithelial cells. Different cell subpopulations configure the epithelial cell monolayers that line the whole intestine lumen: enterocytes and colonocytes specialized in nutrient absorption, Goblet cells specialized in mucin secretion, the major components of mucus, Paneth cells, which release a set of antimicrobial molecules such as defensins (for instance the antimicrobial peptide Reg III $\gamma$ ) and lysozyme, and finally $\mathrm{M}$ cells, which are a kind of cellular mediator between the molecules/microorganisms present in the intestinal lumen and the innate immunity, and which are specialized in transepithelial transport [57]. Interaction of probiotics at this level is very important to keep the barrier function, this being achieved by reinforcing the so-called tight junctions, special locations in which the membranes of two adjacent epithelial cells join together, sealing the lateral surfaces of the enterocytes.

Finally, one of the major points of interaction between probiotics and the human host is the GALT. This is one of the mucosal associated lymphoid tissues of the human body and, in the lower part of the GIT, is composed mainly of Peyer's patches and lymphoid/plasma cells that are diffusely distributed throughout the gut mucosa. Peyer's patches are GALT locations specialized in immune surveillance. In this place antigen-presenting cells, such as dendritic cells or macrophages, interact with $\mathrm{T}$ and $\mathrm{B}$ cells after direct or M-cell-mediated sampling of the intestinal content [58]. 


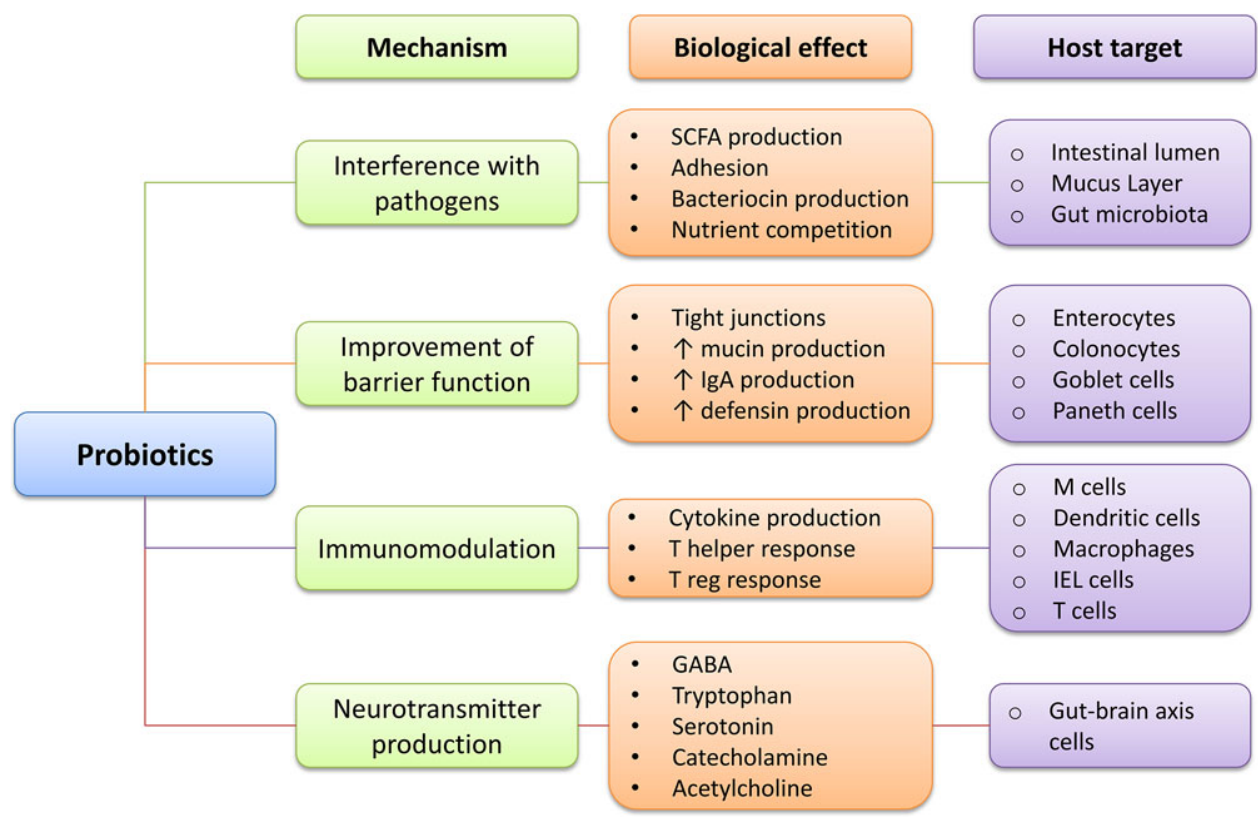

Figure 2. Diagram representing the main mechanisms of action of probiotics. Mechanisms, biological processes, and host cells responsible for the interaction are shown color coded.
Probiotics, as with other bacteria, have a set of relatively wellconserved molecules known as microbial-associated molecular patterns. They interact with specific pattern-recognition receptors present on the surface or in the organelle membrane of epithelial and dendritic cells, this interaction being responsible for not only some of the beneficial effects attributed to probiotics, but also key for the normal interaction of the intestinal microbiota with the human host [59].

Some of the receptor families present on immune and epithelial cells are Toll-like receptors, Nod-like receptors or C-type lectin receptors, and RIG-I-like receptors. Interaction of microbial-associated molecular patterns with patternrecognition receptors determine maturation of antigenpresenting cells, which will subsequently define the type of the immune response developed, which may be mainly effector $\left(\mathrm{T}_{\mathrm{h}} 1, \mathrm{~T}_{\mathrm{h}} 2\right.$, or $\left.\mathrm{T}_{\mathrm{h}} 17\right)$ or regulatory $\left(\mathrm{T}_{\text {reg }}\right)$.

$\mathrm{T}_{\text {reg }}$ response is directly related to the maintenance of intestinal homeostasis and the development of tolerance toward the resident microbiota [60]. Probiotics inducing the $\mathrm{T}_{\text {reg }}$ response are of paramount importance, notably in the framework of IBD and other inflammatory diseases. It has been observed in germ-free animals that colonization with a model microbiota induces both the recruitment and production of $\mathrm{T}_{\text {reg }}$ cells in the intestinal mucosa, being essential to maintain a low inflammation grade in the gut mucosa. Some probiotic bacteria such as members of the genus Lactobacillus may promote this beneficial process through the production of immunomodulatory peptides, such as peptide STp. This peptide acts by inducing an anti-inflammatory cytokine profile in dendritic cells, while downregulating inflammatory mediators, both in healthy controls and UC patients $[61,62]$. Induction of the Treg response has also been observed in some members of the Clostridium clusters IV, XIVa, and XVIII, where some of the strains are claimed to be emerging probiotics [63]. In this case, the homeostatic effect of these bacteria is supported by the production of SCFAs, notably butyrate.

Apart from the direct contact with mucosa, soluble factors synthesized by probotic bacteria are able to stimulate host responses. In this regard, butyrate producers play a key role in the maintenance of the epithelial barrier, since butyrate is one of the main energy sources of enterocytes [64]. Also, a $15 \mathrm{kDa}$ protein secreted by Faecalibacterium prausnitzii is able to inhibit the NF-кB pathway in intestinal epithelial cells and to prevent colitis in an animal model [65]. The reader is prompt to other reviews dealing with the role of soluble factors produced by the intestinal microbiota with a potential role in the molecular cross-talking with the human host [66].

\section{Gut microbiota modulation as a target for dietary interventions using probiotics}

The ultimate goal of a probiotic dietary intervention is to have a positive effect on the health of the target population. This could be achieved through different actions, such as modulation of immunological responses, amelioration of the nutritional state of the host, or those derived from the modulation of the gut microbiota. For many years, it was generally accepted that the majority of the desired probiotic effects were associated with microbial ecological changes in the gut. In this regard, some preclinical studies in animal models have shown that the introduction of Bifidobacterium or Lactobacillus strains in the gut environment of mice, using different animal models, clearly impact on the microbiota composition of the murine gut [67-70]. However, recent literature indicates that these microbiota modifications are not so 
evident in humans, suggesting that bacterial metabolic shifts, rather than a different representation of the microbial populations, could play a pivotal role in the final physiological outcome. In this regard, researchers have taken advantage of novel omics techniques, mainly metagenomics, metatranscriptomics and metabolomics, to unravel the physiological and ecological processes underlying the observed probiotic effects in humans.

One of the most relevant studies was performed by McNulty et al. [71]. In this work, the fecal microbiomes and metatranscriptomes of adult female monozygotic twins were characterized prior to, during and following the consumption of a commercially available fermented milk containing, among other microorganisms, a strain of Bifidobacterium animalis subsp. lactis with attributed health effects. Surprisingly, the bacterial species composition did not significantly change during the course of the study. However, significant changes were detected in the human fecal metatranscriptome during the period of fermented milk consumption, especially those related with the metabolism of plant polysaccharides. Also, a probiotic cheese containing two Lactobacillus strains from the species Lactobacillus rhamnosus and Lactobacillus acidophilus did not significantly modify the levels of the major microbial groups in an elderly population, although some significant changes were observed in minority populations [72]. In a different work, administration of $L$. acidophilus NCFM and B. animalis subsp. lactis Bi-07 did not alter the global composition of the main bacterial groups present in the fecal microbiota of children with atopic dermatitis [73].

In contrast to these observations, other studies have shown that probiotics can cause a shift in specific bacterial populations, with or without associated microbiota metabolic changes. For instance, using high-throughput $16 \mathrm{~S}$ ribosomal RNA gene sequencing, Plaza-Díaz et al. [74] showed that ingestion of L. rhamnosus, Lactobacillus paracasei or Bifidobacterium breve strains by an adult population induced changes in the fecal bacterial population. Also, Lactobacillus salivarius Ls33 was able to modify the fecal bacterial populations of obese adolescents, including several Clostridium groups, although the local concentration of SCFAs remained unaffected [75]. A few works have studied the role of different probiotic interventions in the microbiota of patients with IBS. During intervention with a multispecies probiotic supplementation in IBS patients, the probiotic group showed fluctuations in some clostridia populations that were stably maintained during the intervention and these alterations were correlated with the alleviation of gastrointestinal symptoms [76]. Also, fermented milk containing $B$. animalis subsp. lactis was able to decrease the abundance of some harmful bacteria and modify the colonic SCFAs production [77]. Furthermore, the effects of probiotic fermented milk on the fecal metabolome of adult patients with IBS was evaluated through a clinical trial, but no difference in the fecal metabolites of the patients in the probiotic groups was detected [78].

Probiotics in combination with other active dietary components also represent a very remarkable strategy to bene- ficially affect host health. In this regard, synbiotics, that is probitotic and prebiotic combinations, have been used [79]. Some of these synbiotics do not affect microbiota composition, but are able to modify its metabolic profile. For instance, synbiotic L. acidophilus NCFM and cellobiose does not affect human gut bacterial diversity but increased the local concentration of branched-chain fatty acids, which play a role in the maintenance of gut physiology [80].

These studies highlight the need to use complementary approaches to provide the necessary observations that could explain the molecular principles underlying the probiotic effect. The majority of the probiotic intervention studies involving next-generation sequencing (NGS) approaches and/or metabolomics have shown that no relevant changes in the gut microbiota structure are detected during or after the intervention. However, we have to bear in mind that the effect is strain specific, and may vary depending on the physiology of the individuals and the dietary context in which the interventions were performed [81]. Furthermore, there is clear evidence that a shift in the local gut environment does not necessarily involve changes in the bacterial populations and, under some circumstances, probiotic-driven effects seem to be related to a metabolic shift of the gut microbiota (for instance, increase in SCFA production), rather than to a change of the microbiota profile.

Interestingly probiotics develop relevant roles in terms of cross-feeding activities when they enter in contact with the rest of microbial communities, influencing the metabolic capacities of other commensal members. Great examples of this can be found within the genus Bifidobacterium, which are important gut commensals with some probiotic representatives. Bifidobacteria are able to metabolize different carbohydrates originated from diet or from the host mucosa, producing different proportions of acetic/lactic acid through their specific fermentative pathway [82]. Presence of a given Bifidobacterium species may influence the metabolic activity of other species through cross-feeding mechanisms and vice versa, as it was observed in cocultures of different bifidobacteria species. On the one hand, Bifidobacterium bifidum increases its metabolic activity when cocultured in the presence of other species such as $B$. breve [83]. On the other hand, $B$. breve benefits from the sialic acid released from the B. bifidum metabolism on host glycans, such as human milk oligosaccharides (more concisely 3 -sialyllactose) or mucin $[84,85]$. This is a good example on how bifidobacteria administered as probiotics may affect the metabolic capacity of commensal gut populations by affecting the way in which host glycans are metabolized. Further, changes in the production of organic acids will affect the metabolism of other microbial groups, such as butyrateproducing bacteria [86].

Unlike gut microbiota, less attention has been paid to the gut virome. It has been postulated that the number of unique genes coding in the virome may be immense, as it includes not only those that infect cells, but bacteriophages, retroviruses, fungi viruses etc. [87]. Bacteriophages, or viruses that infect commensal bacteria, are important means for 
microbiota modulation and they have been proposed to be used for microbiota modulation by targeting specific commensal groups, which may have a profound impact on the immune response at the mucosa level [88]. For all these reasons further research will likely elucidate some of the effects of the gut virome not only in host disease, but as beneficial modulators of gut physiology.

In this regard some probiotic interventions show a pivotal role in the metabolic activity of intestinal microbiota. One of the most relevant effects is the increase in intestinal butyrate concentrations or butyrate-producing bacteria. A recent study has shown that food-allergic infants fed with a L. rhamnosus GG-supplemented formula increased the butyrate-producing bacterial population in feces [89]. Also, Ferrario et al. [90] showed an increase of some butyrate produces after intake of a strain of L. paracasei, and suggest that fecal butyrate concentrations could represent an important biomarker for identifying subjects who may benefit from probiotic treatment.

\section{Can we associate beneficial effects to specific microbes/microbial populations?}

Attempts have been made to define the microbial taxa that constitute a healthy human gut microbiota. However, its composition is highly variable between different individuals due to the strong influence that host and environmental factors exert on the gut microbial ecosystem, making it extremely difficult to establish a common "human core microbiome" [91]. Thus, at the moment, the scientific community has not reached a consensus to define our beneficial intestinal microbial fingerprint. However, accumulating observational studies have shown that there is a correlation between some bacterial populations in the human gut microbiota and different physiological states, including those having an influence on health. One of the typical examples of this correlation is that of bifidobacteria in breast-fed infants. Breast-feeding constitutes the golden standard for infant nutrition and numerous studies have shown its protective effect on immunological disorders and infections [92]. Therefore, since bifidobacteria populations are abundant in breast-fed infants, compared with those fed with infant formula, it is generally believed that the presence of bifidobacteria in the microbiota of infants is an indicator of good health [93, 94]. On the other hand, currently we know that aberrancies in the gut microbiota composition are associated with several diseases, including chronic diseases such as type 2 diabetes or Crohn's disease [95, 96], immune-related disorders [97]. Consistently, metagenomic studies have shown that specific groups of strict gut anaerobes are underrepresented in disease states, such as F. prausnitzii and other members of the Ruminococcaceae family in Crohn's disease [96, 98], or representatives of the Clostridium XIVa cluster, including members of the Lachnospiraceae family, in a myriad of diseases [97-99].
Diseases in which the strict anaerobic population is decreased normally involve physiological conditions in which inflammation and oxidative stress are present [100]. Unfortunately, these "most wanted" taxa, those highly prevalent and abundant in the human microbiota that appear to be associated with a healthy state, or those that are depleted in physiological disorders and/or diseases, are normally fastidious or non-culturable anaerobes that do not grow using traditional cultivation methods. This means that we know of their existence and identity mainly because of the advent and refinement of NGS methodologies, but we know very little about their functions. Even using advanced massive sequencing methods, some microbial groups (e.g. Archaea) could pass totally unnoticed [101]. Therefore, in practice, the physiology and metabolic capabilities of the majority of the "healthy" microbial representatives of our gut have not been studied yet, with a few exceptions, such as Bifidobacterium and Lactobacillus strains, which grow relatively well under laboratory conditions, or F. prausnitzii, one of the "emerging probiotics" [102]. Thus, a pivotal activity in future probiotic research will be to develop novel microbial cultivation strategies of the nonculturable "potential" beneficial microbes that are associated with healthy conditions in humans, either alone or cocultivated in defined microbial consortia [103], which will be critical for the food and pharma industry in order to be able to perform disease-targeted probiotic interventions and bring novel products to the market.

\section{Bioinformatics gives the meaning}

A way to investigate gut microbiota modulation is highthroughput sequencing of the genes or a precise gene fragment, usually a variable region of the 16 rDNA gene. Metagenomes are an analytically challenging type of genomic data, which encompass sequences of all genes from the totality of a complex microbial community. Although NGS allows the genomic analysis of samples obtained directly from the environment, such an approach generates huge volumes of data and demands a powerful means of analysis. Many gut metagenomic studies still perform 16S rRNA sequencing, but this approach do not allow monitoring changes in the minor players of the gut microbiota [101]. For this reason whole shotgun sequencing is becoming the technology of choice to perform sequence analysis and community comparison. Either way, bioinformatics is a critical factor in these studies (Fig. 3).

In terms of 16S rRNA gene analyses, the Genboree Microbiome Toolset supports community profiling (i.e. determination of the abundance of each type of microbe) [104], QIIME [105], and Mothur [106] (also part of Genboree) can be used to obtain quantitative insights into microbial ecology, BLAST [107] and Cd-hit [108] facilitate the comparison of large sets of proteins, and the Chimera Slayer is used to detect sequences falsely interpreted as organisms (contributing to false perceptions of sample diversity and the false 


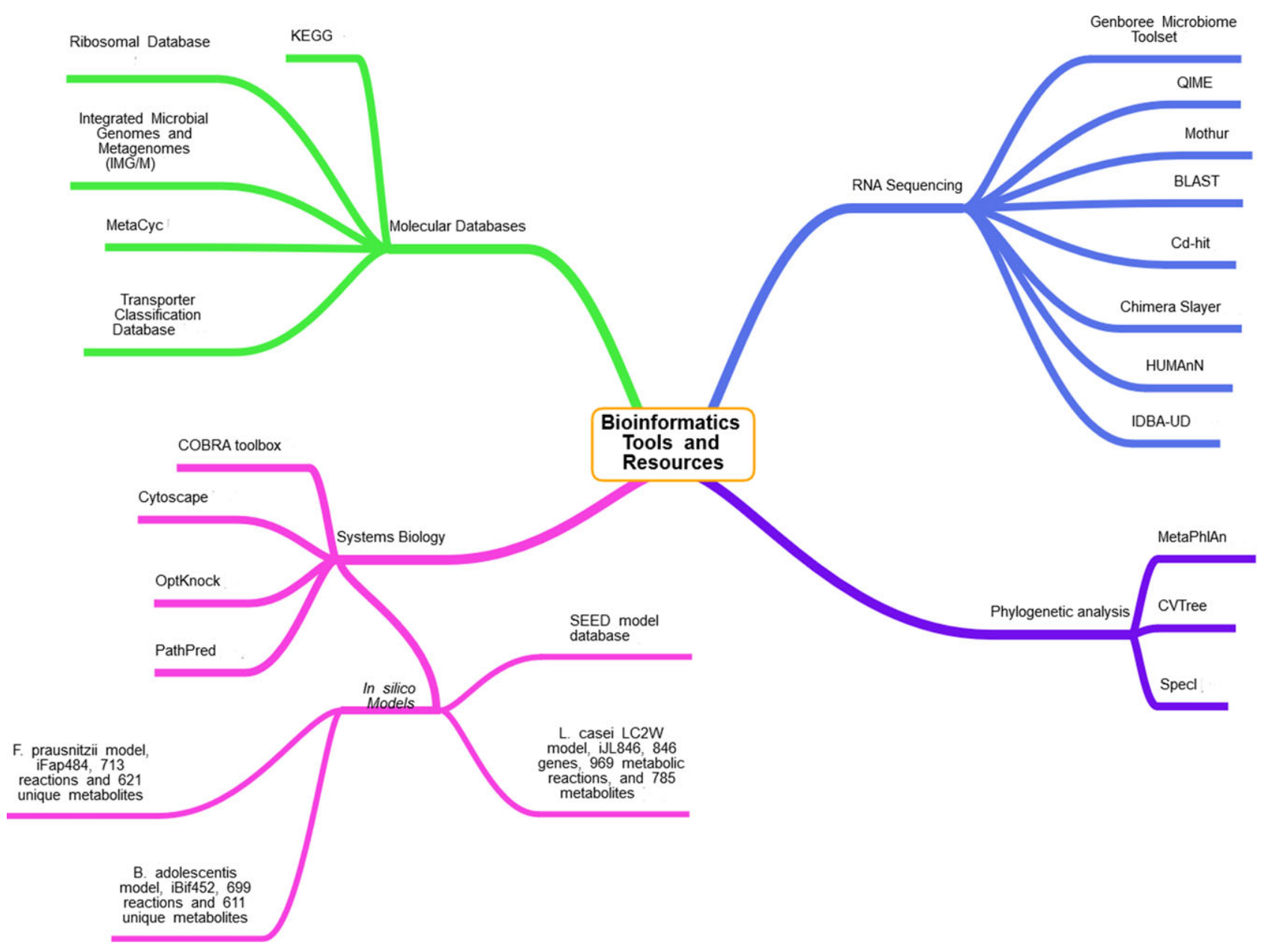

Figure 3. Schematic overview of the bioinformatics tools available for the study of probiotic and microbiota functionality, including molecular databases, system biology tools, or in silico models of metabolic microorganism among others.

identification of novel taxa) [109]. Furthermore, the Ribosomal Database Project classifiers may help in the assignment of rRNA sequences into bacterial taxonomy [110].

In whole genome studies, there is also a set of commonly used tools. Bowtie2 tool [111] enables read alignment to the human genome (reference) and to the KEGG database [112]. KEGG alignment results can be further analyzed by the HUMAnN system to determine the relative abundance of the gut microbial functional pathways [113]. For example, linear discriminant analysis may help in identifying enzymatic pathways that are significantly different between pre- and postintervention [114].

IDBA-UD is utilized in de novo assembly of metagenomic sequencing data [115]. Genes are clustered based on their abundance data using the Canopy-based algorithm and the profiles of these groups may be calculated as the samplewise median gene abundance [116]. Often, the HMP DACC database supports the comparison of assemblies and reference genomes, performed using MUMmer3.0 [117] or a similar tool. In turn, phylogenetic analysis is supported by tools such as the CVTree server, which implements a whole genome-based, alignment-free composition vector method [118]; MetaPhlAn, which estimates the abundance of species in each sample according to the number of mapped reads to its markers [119]; and, SpecI, which is a method of grouping organisms into species clusters based on phylogenetic marker genes [120]. These methods have been used, for instance, to analyze how species within the same genome interaction groups decrease or increase their abundance together during the dietary interventions [121].

The restoration or strengthening of the intestinal microbiota through diet-based approaches such as probiotics has been proposed as a means to enhance relevant functions of the GIT. Under this scenario, systems biology methods and tools provide an invaluable research workbench to understand and model the metabolism and modulation of intestinal microbiota. Arguably, the reconstruction of genome-scale metabolic models and the elucidation of interaction networks are the two most prominent contributions here.

Databases such as KEGG [122], Metacyc [123], Model SEED [124] and Transporter Classification Database (TCDB) 
[125] are commonly used in metagenome reconstruction projects, while the OptKnock algorithm [126] and the COBRA toolbox [127] facilitate flux balance analysis and additional metabolic profiling. For example, the analysis of a community level metabolic network model supported the discovery of shifts associated with obesity and IBD [128]. The reconstruction of a genome-scale metabolic model for L. casei LC2W enabled the identification of essential amino acids and vitamins and the exploration of the biosynthetic potential of some metabolites [129]. The genome-scale metabolic reconstruction of Bifidobacterium adolescentis L2-32 and F. prausnitzii A2-165 enabled in silico simulation of the metabolic crosstalk between the two species and evidenced the importance of acetate supply into butyrate production [130]. Additional details on up-to-date genome-scale models of the human gut microbiome may be found in ref. 131 .

Computational pathway analysis, facilitated by tools such as PathPred [132], is being utilized in the study of community level biotransformation. For example, a metametabolome network covering Bacteroidetes, Firmicutes, and Actinobacteria brought into light cross-feeding relationships between some gut microbe enzymes and host carbohydrate metabolism enzymes [133]. Besides assessing hostmicrobe symbiosis, pathway analysis offered interesting insights into diet modulation and the use of probiotics in treating metabolic disorders. Likewise, Boolean network modeling and dynamic analysis enable the inference of important relationships within gut microbiota composition. More specifically, this methodology was used to explore the dynamics of clindamycin antibiotic treatment in $C$. difficile infection and to predict therapeutic probiotic interventions to suppress $C$. difficile infection [134].

The analysis of the interaction of bacterial genes and dietary components is another valuable alternative. For example, a protein-protein interaction network supported the study of potential dietary interventions targeting the SCFA metabolism, namely topological metrics enabled the identification of the most vulnerable protein targets of the butyrate and propionate metabolic pathways (i.e. protein targets are more likely to change gene expression activity) [135]. Within network-based studies, Cytoscape is the most used platform to visualize and analyze interaction networks [136]. Besides providing generic means of visualization and topological exploration, this platform has a number of different applications to enable multiscale data integration, data clustering, enrichment analysis, and network comparison, among others.

In conclusion, bioinformatics and systems biology build an excellent platform to generate theoretical models to predict how interventions with probiotics, bioactive compounds, or specific diets can shape the structure and function of the gut microbiota, thus inducing the desired host responses under specific physiological conditions. They offer a range of possibilities to develop novel microbial cultivation strategies of "potential" beneficial microbes, which are associated with healthy conditions in humans. Bioinformatics could also allow the development of novel tailor-made substrates that facilitate the enrichment of the beneficial bacteria within a complex microbiota, through prediction of the nutritional and environmental needs of these bacteria, among other potential applications. However, until now, the physiological validation of these theoretical models is still weak, and need to be addressed more deeply in order to be able to perform novel disease-targeted probiotic intervention strategies.

\section{Conclusions and future perspectives in probiotic research}

Research on human diseases is revealing the vital roles played by the gut microbiota. This interaction is not restricted to the intestinal mucosa, but to distant locations such as the gutbrain axis. Understanding the impact of the gut microbiota on the host health is essential to design strategies focused in their manipulation, notably in cases where altered microbial patterns are present. Probiotics are important means to produce specific host benefits through different mechanisms, among which modification of the intestinal microbiota is noteworthy. However, recent literature suggests that the modification of the intestinal microbiota is not strictly necessary to produce a beneficial effect [137]. In fact, the probiotic effect could be triggered via an interaction with the immune system, which does not necessarily mean a modification of the local microbial environment. In relation to this, during the last years, it is becoming clear that some strains of the most commonly used probiotics (belonging to the genera Lactobacillus and Bifidobacterium) do not colonize the gut and are released from the intestine after the probiotic intervention; thus, they could not be considered stable members of the intestinal microbiota. Therefore, for future probiotic research, we consider of key importance promoting studies on commensal intestinal microorganisms, well adapted to the gut environment, that are able to modify the local microbial ecosystem, thus potentially generating long-term effects. However, the industrial exploitation of novel commensal strains belonging to nonconventional probiotic genera (i.e. Faecalibacterium, Akkermansia, or Eubacterium) could be hampered by the lack an appropriate regulatory frame and safety studies.

On the other hand, in spite of our knowledge on the molecular mechanisms underlying some of the beneficial effects of probiotics, we are far of clinically prove their efficacy in many inflammatory and autoimmune diseases. In the future, technological improvements for commensal gut microorganism culture, coculture, and administration, together with safety and clinical studies, will provide us with a new generation of probiotics that are currently designed as "emerging." Moreover, knowledge on the molecules responsible for the probiotic effect will allow us to design new strategies allowing culture, coculturing procedures, and propagation of these often fastidious and oxygen-sensitive bacteria. 
Research in our lab is funded by Grants AGL2013-44039$R$ and AGL2013-44761-P from the Spanish "Plan Estatal de $I+D+I$." Part of the authors is also partially funded by the [15VI013] Contract-Programme from the University of Vigo and the Agrupamento INBIOMED from DXPCTSUG-FEDER unha maneira de facer Europa (2012/273). B. S. was recipient of a Ramón y Cajal postdoctoral contract from the Spanish Ministry of Economy and Competitiveness.

The authors have declared no conflict of interest.

\section{References}

[1] Hooper, L. V., Gordon, J. I., Commensal host-bacterial relationships in the gut. Science 2001, 292, 1115-1118.

[2] Sommer, F., Bäckhed, F., The gut microbiota - masters of host development and physiology. Nat. Rev. Microbiol. 2013, 11, 227-238.

[3] Al-Asmakh, M., Zadjali, F., Use of germ-free animal models in microbiota-related research. J. Microbiol. Biotechnol. 2015, 25, 1583-1588.

[4] Deplancke, B., Gaskins, H. R., Microbial modulation of innate defense: goblet cells and the intestinal mucus layer. Am. J. Clin. Nutr. 2001, 73, 1131S-1141S.

[5] Macpherson, A. J., Harris, N. L., Interactions between commensal intestinal bacteria and the immune system. Nat. Rev. Immunol. 2004, 4, 478-485.

[6] Huttenhower, C., Gevers, D., Knight, R., Abubucker, S. et al., Structure, function and diversity of the healthy human microbiome. Nature 2012, 486, 207-214.

[7] Methé, B. A., Nelson, K. E., Pop, M., Creasy, H. H. et al., A framework for human microbiome research. Nature 2012, $486,215-221$.

[8] Ding, T., Schloss, P. D., Dynamics and associations of microbial community types across the human body. Nature 2014, 509, 357-360.

[9] Qin, J., Li, R., Raes, J., Arumugam, M. et al., A human gut microbial gene catalogue established by metagenomic sequencing. Nature 2010, 464, 59-65.

[10] Costello, E. E. K., Lauber, C. C. L., Hamady, M., Fierer, N. et al., Bacterial community variation in human body habitats across space and time. Science (80-) 2009, 326, 16941697.

[11] Sender, R., Fuchs, S., Milo, R., Are we really vastly outnumbered? Revisiting the ratio of bacterial to host cells in humans. Cell 2016, 164, 337-340.

[12] Ley, R. E., Peterson, D. A., Gordon, J. I., Ecological and evolutionary forces shaping microbial diversity in the human intestine. Cell 2006, 124, 837-848.

[13] Eckburg, P. B., Bik, E. M., Bernstein, C. N., Purdom, E. et al., Diversity of the human intestinal microbial flora. Science 2005, 308, 1635-1638.

[14] Tannock, G. W., What immunologists should know about bacterial communities of the human bowel. Semin. Immunol. 2007, 19, 94-105.
[15] Van den Abbeele, P., Belzer, C., Goossens, M., Kleerebezem, M. et al., Butyrate-producing Clostridium cluster $X I V a$ species specifically colonize mucins in an in vitro gut model. ISME J. 2013, 7, 949-961.

[16] Arumugam, M., Raes, J., Pelletier, E., Le Paslier, D. et al., Enterotypes of the human gut microbiome. Nature 2011, $12,174-180$.

[17] Jeffery, I. B., Claesson, M. J., O'Toole, P. W., Shanahan, F., Categorization of the gut microbiota: enterotypes or gradients? Nat. Rev. Microbiol. 2012, 10, 591-592.

[18] Wu, G. D., Chen, J., Hoffmann, C., Bittinger, K. et al., Linking long-term dietary patterns with gut microbial enterotypes. Science 2011, 334, 105-108.

[19] Huse, S. M., Ye, Y., Zhou, Y., Fodor, A. A., A core human microbiome as viewed through $16 \mathrm{~S}$ rRNA sequence clusters. PLoS One 2012, 7, e34242.

[20] Wu, G. D., Chen, J., Hoffmann, C., Bittinger, K. et al., NIH Public Access. Science (80-) 2012, 334, 105-108.

[21] Knights, D., Ward, T. L., McKinlay, C. E., Miller, H. et al., Rethinking enterotypes. Cell Host Microbe 2014, 16, 433437.

[22] Schrezenmeir, J., de Vrese, M., Probiotics, prebiotics, and synbiotics-approaching a definition. Am. J. Clin. Nutr. 2001, 73, 361S-364S.

[23] Gordon, S., Elie Metchnikoff: father of natural immunity. Eur. J. Immunol. 2008, 38, 3257-3264.

[24] Lilly, D. M., Stillwell, R. H., Probiotics: growth-promoting factors produced by microorganisms. Science (80-). 1965, 147, 747-748.

[25] Parker, R. B., Probiotics, the other half of the antibiotic history. Anim. Nutr. Health 1974, 29, 4-8.

[26] Fuller, R., Probiotics in man and animals. J. Appl. Bacteriol. $1989,66,365-378$.

[27] Havenaar, R., Huis In't Veld, J. H. J., Probiotics: a general view. Lact. Acid Bact. 1992, 1, 151-170.

[28] Salminen, S., Uniqueness of probiotic strains. IDF Nutr. News Lett. 1995, 5, 16-18.

[29] Schaafsma, G., State of art concerning probiotic strains in milk products. IDF Nutr. News Lett. 1996, 5, 23-24.

[30] Salminen, S., Bouley, C., Boutron-Ruault, M. C., Cummings, J. H. et al., Functional food science and gastrointestinal physiology and function. Br. J. Nutr. 1998, 80 (Supp/ 1), S147-S171.

[31] ILSI, Scientific concepts of functional foods in Europe. Consensus document. Br. J. Nutr. 1999, 81 (Supp/ 1), S1-S27.

[32] Salminen, S., Ouwehand, A., Benno, Y., Lee, Y. K., Probiotics: how should they be defined? Trends Food Sci. Technol. 1999, 10, 107-110.

[33] Ouwehand, A. C., Salminen, S. J., The health effects of cultured milk products with viable and non-viable bacteria. Int. Dairy J. 1998, 8, 749-758.

[34] Jijon, H., Backer, J., Diaz, H., Yeung, H. et al., DNA from probiotic bacteria modulates murine and human epithelial and immune function. Gastroenterology 2004, 126, 13581373. 
[35] FAO, Probiotics in food. Food Nutr. Pap. 2001, 85, 71.

[36] Hill, C., Guarner, F., Reid, G., Gibson, G. R. et al., Expert consensus document: The International Scientific Association for Probiotics and Prebiotics consensus statement on the scope and appropriate use of the term probiotic. Nat. Rev. Gastroenterol. Hepatol. 2014, 11, 506-514.

[37] Ritchie, M. L., Romanuk, T. N., A meta-analysis of probiotic efficacy for gastrointestinal diseases. PLoS One 2012, 7.

[38] Allen, S. J., Martinez, E. G., Gregorio, G. V., Dans, L. F. et al., Probiotics for treating acute infectious diarrhoea. Cochrane Database Syst. Rev. 2010, 11, CD003048.

[39] McFarland, L. V., Meta-analysis of probiotics for the prevention of traveler's diarrhea. Travel Med. Infect. Dis. 2007, $5,97-105$.

[40] Szajewska, H., Guarino, A., Hojsak, I., Indrio, F. et al., Use of probiotics for management of acute gastroenteritis: a position paper by the ESPGHAN Working Group for Probiotics and Prebiotics. J. Pediatr. Gastroenterol. Nutr. 2014, $58,531-539$.

[41] Fiocchi, A., Pawankar, R., Cuello-Garcia, C., Ahn, K. et al., World Allergy Organization-McMaster University Guidelines for Allergic Disease Prevention (GLAD-P): probiotics. World Allergy Organ. J. 2015, 8, 4.

[42] Szajewska, H., Kołodziej, M., Systematic review with metaanalysis: Saccharomyces boulardii in the prevention of antibiotic-associated diarrhoea. Aliment. Pharmacol. Ther. 2015, 42, 793-801.

[43] Szajewska, H., Kołodziej, M., Systematic review with metaanalysis: Lactobacillus rhamnosus GG in the prevention of antibiotic-associated diarrhoea in children and adults. Aliment. Pharmacol. Ther. 2015, 42, 1149-1157.

[44] Szajewska, H., Canani, R. B., Guarino, A., Hojsak, I. et al., Probiotics for the prevention of antibiotic-associated diarrhea in children. J. Pediatr. Gastroenterol. Nutr. 2016, 62, 495-506.

[45] Hevia, A., Delgado, S., Margolles, A., Sánchez, B., Application of density gradient for the isolation of the fecal microbial stool component and the potential use thereof. Sci. Rep. 2015, 5, 16807.

[46] Elahi, B., Nikfar, S., Derakhshani, S., Vafaie, M. et al., On the benefit of probiotics in the management of pouchitis in patients underwent ileal pouch anal anastomosis: a metaanalysis of controlled clinical trials. Dig. Dis. Sci. 2008, 53, 1278-1284.

[47] Marteau, P., Sokol, H., Dray, X., Seksik, P., Bacteriotherapy for inflammatory bowel disease: therapeutic tool and/or pharmacological vectors? Gastroentérol. Clin. Biol. 2009, 33 (Supp/ 3), S228-S234.

[48] Moayyedi, P., Ford, A. C., Talley, N. J., Cremonini, F. et al., The efficacy of probiotics in the treatment of irritable bowel syndrome: a systematic review. Gut 2010, 59, 325332.

[49] Spiller, R., Review article: probiotics and prebiotics in irritable bowel syndrome. Aliment. Pharmacol. Ther. 2008, 28, 385-396.
[50] Lesbros-Pantoflickova, D., Corthésy-Theulaz, I., Blum, A. L., Helicobacter pylori and probiotics. J. Nutr. 2007, 137, 812S$818 S$.

[51] Homan, M., Orel, R., Are probiotics useful in Helicobacter pylori eradication? World J. Gastroenterol. 2015, 21, 1064410653.

[52] de Vrese, M., Stegelmann, A., Richter, B., Fenselau, S. et al., Probiotics-compensation for lactase insufficiency. Am. J. Clin. Nutr. 2001, 73, 421S-429S.

[53] Sánchez, B., De Los Reyes-Gavilan, C. G., Margolles, A., Gueimonde, M., Probiotic fermented milks: present and future. Int. J. Dairy Technol. 2009, 62, 472-483.

[54] Tojo, R., Suárez, A., Clemente, M. G., De Los Reyes-Gavilán, C. G. et al., Intestinal microbiota in health and disease: Role of bifidobacteria in gut homeostasis. World J. Gastroenterol. 2014, 20, 15163-15176.

[55] Maynard, C. L., Elson, C. O., Hatton, R. D., Weaver, C. T., Reciprocal interactions of the intestinal microbiota and immune system. Nature 2012, 489, 231-241.

[56] Johansson, M. E. V., Larsson, J. M. H., Hansson, G. C., The two mucus layers of colon are organized by the MUC2 mucin, whereas the outer layer is a legislator of hostmicrobial interactions. Proc. Natl. Acad. Sci. U S A 2011, 108 (Suppl), 4659-4665.

[57] Goto, Y., Ivanov, I. I., Intestinal epithelial cells as mediators of the commensal-host immune crosstalk. Immunol. Cell Biol. 2013, 91, 204-214.

[58] Kamada, N., Seo, S.-U., Chen, G. Y., Núñez, G., Role of the gut microbiota in immunity and inflammatory disease. Nat. Rev. Immunol. 2013, 13, 321-335.

[59] Bouskra, D., Brézillon, C., Bérard, M., Werts, C. et al., Lymphoid tissue genesis induced by commensals through NOD1 regulates intestinal homeostasis. Nature 2008, 456, 507-510.

[60] Wang, Y., Begum-Haque, S., Telesford, K. M., OchoaRepáraz, J. et al., A commensal bacterial product elicits and modulates migratory capacity of $\mathrm{CD} 39(+) \mathrm{CD} 4 \mathrm{~T}$ regulatory subsets in the suppression of neuroinflammation. Gut Microbes 2014, 5, 552-561.

[61] Bernardo, D., Sánchez, B., Al-Hassi, H. O., Mann, E. R. et al., Microbiota/host crosstalk biomarkers: regulatory response of human intestinal dendritic cells exposed to Lactobacillus extracellular encrypted peptide. PLoS One 2012, 7, 1-8.

[62] Al-Hassi, H. O., Mann, E. R., Sanchez, B., English, N. R. et al., Altered human gut dendritic cell properties in ulcerative colitis are reversed by Lactobacillus plantarum extracellular encrypted peptide STp. Mol. Nutr. Food Res. 2014, 58, 1132-1143.

[63] Atarashi, K., Tanoue, T., Oshima, K., Suda, W. et al., Treg induction by a rationally selected mixture of Clostridia strains from the human microbiota. Nature 2013, 500, 232236.

[64] Louis, P., Hold, G. L., Flint, H. J., The gut microbiota, bacterial metabolites and colorectal cancer. Nat. Rev. Microbiol. $2014,12,661-672$. 
[65] Quévrain, E., Maubert, M. A., Michon, C., Chain, F. et al., Identification of an anti-inflammatory protein from Faecalibacterium prausnitzii, a commensal bacterium deficient in Crohn's disease. Gut 2015, 65, 415-425.

[66] Ruiz, L., Hevia, A., Bernardo, D., Margolles, A. et al., Extracellular molecular effectors mediating probiotic attributes. FEMS Microbiol. Lett. 2014, 359, 1-11.

[67] Arthur, J. C., Gharaibeh, R. Z., Urines, J. M., Perez-Chanona, E. et al., VSL\#3 probiotic modifies mucosal microbial composition but does not reduce colitis-associated colorectal cancer. Sci. Rep. 2013, 3, 2868.

[68] Sugahara, H., Odamaki, T., Fukuda, S., Kato, T. et al., Probiotic Bifidobacterium longum alters gut luminal metabolism through modification of the gut microbial community. Nature 2015, 5, 1-11.

[69] Turroni, F., Milani, C., Duranti, S., Mancabelli, L. et al., Deciphering bifidobacterial-mediated metabolic interactions and their impact on gut microbiota by a multi-omics approach. ISME J. 2016, 10, 1656-1668.

[70] Wang, W.-L., Xu, S.-Y., Ren, Z.-G., Tao, L. et al., Application of metagenomics in the human gut microbiome. World $\mathrm{J}$. Gastroenterol. 2015, 21, 803-814.

[71] McNulty, N. P., Yatsunenko, T., Hsiao, A., Faith, J. J. et al., The impact of a consortium of fermented milk strains on the gut microbiome of gnotobiotic mice and monozygotic twins. Sci. Transl. Med. 2011, 3, 106ra106.

[72] Lahtinen, S. J., Forssten, S., Aakko, J., Granlund, L. et al., Probiotic cheese containing Lactobacillus rhamnosus HN001 and Lactobacillus acidophilus NCFM ${ }^{\top}$ modifies subpopulations of fecal lactobacilli and Clostridium difficile in the elderly. Age 2012, 34, 133-143.

[73] Nadja, L., Vogensen, F. K., Gøbe, R., Michaelsen, K. F. et al., Predominant genera of fecal microbiota in children with atopic dermatitis are not altered by intake of probiotic bacteria Lactobacillus acidophilusNCFM andBiфdobacterium animalis subsp.lactisBi-07. FEMS Microbiol. Ecol. 2011, 75, 482-496.

[74] Plaza-Díaz, J., Fernández-Caballero, J. Á., Chueca, N., García, F. et al., Pyrosequencing analysis reveals changes in intestinal microbiota of healthy adults who received a daily dose of immunomodulatory probiotic strains. Nutrients 2015, 7, 3999-4015.

[75] Larsen, N., Vogensen, F. K., Gøbel, R. J., Michaelsen, K. F. et al., Effect of Lactobacillus salivarius Ls-33 on fecal microbiota in obese adolescents. Clin. Nutr. 2013, 32, 935940.

[76] Lyra, A., Krogius-Kurikka, L., Nikkilä, J., Malinen, E. et al., Effect of a multispecies probiotic supplement on quantity of irritable bowel syndrome-related intestinal microbial phylotypes. BMC Gastroenterol. 2010, 10, 110.

[77] Veiga, P., Pons, N., Agrawal, A., Oozeer, R. et al., Changes of the human gut microbiome induced by a fermented milk product. Sci. Rep. 2014, 4, 1-9.

[78] Hong, Y.-S., Hong, K. S., Park, M.-H., Ahn, Y.-T. et al., Metabonomic understanding of probiotic effects in humans with irritable bowel syndrome. J. Clin. Gastroenterol. 2011, $45,415-425$
[79] Patel, R., Dupont, H. L., New approaches for bacteriotherapy: prebiotics, new-generation probiotics, and synbiotics. Clin. Infect. Dis. 2015, 60, S108-S121.

[80] van Zanten, G. C., Krych, L., Röytiö, H., Forssten, S. et al., Synbiotic Lactobacillus acidophilus NCFM and cellobiose does not affect human gut bacterial diversity but increases abundance of lactobacilli, bifidobacteria and branchedchain fatty acids: a randomized, double-blinded cross-over trial. FEMS Microbiol. Ecol. 2014, 90, 225-236.

[81] Derrien, M., van Hylckama Vlieg, J. E., Fate, activity, and impact of ingested bacteria within the human gut microbiota. Trends Microbiol. 2015, 23, 354-366.

[82] Sánchez, B., Noriega, L., Ruas-Madiedo, P., de los ReyesGavilán, C. G. et al., Acquired resistance to bile increases fructose-6-phosphate phosphoketolase activity in Bifidobacterium. FEMS Microbiol. Lett. 2004, 235, 35-41.

[83] Turroni, F., Özcan, E., Milani, C., Mancabelli, L. et al., Glycan cross-feeding activities between bifidobacteria under in vitro conditions. Front. Microbiol. 2015, 6, 1030.

[84] Egan, M., Motherway, M. O., Kilcoyne, M., Kane, M. et al., Cross-feeding by Bifidobacterium breve UCC2003 during co-cultivation with Bifidobacterium bifidum PRL2010 in a mucin-based medium. BMC Microbiol. 2014, 14, 282.

[85] Egan, M., Motherway, M. O. C., Ventura, M., van Sinderen, D., Metabolism of sialic acid by Bifidobacterium breve UCC2003. Appl. Environ. Microbiol. 2014, 80, 4414-4426.

[86] Rios-Covian, D., Gueimonde, M., Duncan, S. H., Flint, H. J. et al., Enhanced butyrate formation by cross-feeding between Faecalibacterium prausnitzii and Bifidobacterium adolescentis. FEMS Microbiol. Lett. 2015, 362, fnv176.

[87] Cadwell, K., Expanding the role of the virome: commensalism in the gut. J. Virol. 2015, 89, 1951-1953.

[88] Duerkop, B. A., Hooper, L. V., Resident viruses and their interactions with the immune system. Nat. Immunol. 2013, 14, 654-659.

[89] Berni Canani, R., Sangwan, N., Stefka, A. T., Nocerino, R. et al., Lactobacillus rhamnosus GG-supplemented formula expands butyrate-producing bacterial strains in food allergic infants. ISME J. 2015, 10, 1-9.

[90] Ferrario, C., Taverniti, V., Milani, C., Fiore, W. et al., Modulation of fecal Clostridiales bacteria and butyrate by probiotic intervention with Lactobacillus paracasei DG varies among healthy adults. J. Nutr. 2014, 144, 1787-1796.

[91] Caporaso, J. G., Lauber, C. L., Costello, E. K., Berg-Lyons, D. et al., Moving pictures of the human microbiome. Genome Biol. 2011, 12, R50.

[92] Andreas, N. J., Kampmann, B., Le-Doare, M. K., Human breast milk: a review on its composition and bioactivity. Early Hum. Dev. 2015, 91, 629-635.

[93] Arboleya, S., Ruas-Madiedo, P., Margolles, A., Solís, G. et al., Characterization and in vitro properties of potentially probiotic Bifidobacterium strains isolated from breast-milk. Int. J. Food Microbiol. 2011, 149, 28-36.

[94] Turroni, F., Peano, C., Pass, D. A., Foroni, E. et al., Diversity of bifidobacteria within the infant gut microbiota. PLoS One 2012, 7, e36957. 
[95] Qin, J., Li, Y., Cai, Z., Li, S. et al., A metagenome-wide association study of gut microbiota in type 2 diabetes. Nature 2012, 490, 55-60.

[96] Joossens, M., Huys, G., Cnockaert, M., Preter, V. D. et al., Dysbiosis of the faecal microbiota in patients with Crohn's disease and their unaffected relatives. Gut 2011, 60, 631637.

[97] Hevia, A., Milani, C., López, P., Cuervo, A. et al., Intestinal dysbiosis associated with systemic lupus erythematosus. MBio 2014, 5, e01548-e01514.

[98] Gevers, D., Kugathasan, S., Denson, L. A., Vázquez-Baeza, Y. et al., The treatment-naive microbiome in new-onset Crohn's Disease. Cell Host Microbe 2014, 15, 382-392.

[99] Machiels, K., Sabino, J., Vandermosten, L., Joossens, M. et al., Specific members of the predominant gut microbiota predict pouchitis following colectomy and IPAA in UC. Gut 2015, doi: 10.1136/gutjnl-2015-309398.

[100] Morgan, X. C., Tickle, T. L., Sokol, H., Gevers, D. et al., Dysfunction of the intestinal microbiome in inflammatory bowel disease and treatment. Genome Biol. 2012, 13, R79.

[101] Milani, C., Hevia, A., Foroni, E., Duranti, S. et al., Assessing the fecal microbiota: an optimized ion torrent 165 rRNA gene-based analysis protocol. PLoS One 2013, 8, e68739.

[102] Miquel, S., Martín, R., Rossi, O., Bermúdez-Humarán, L. G. et al., Faecalibacterium prausnitzii and human intestinal health. Curr. Opin. Microbiol. 2013, 16, 255-261.

[103] Petrof, E. O., Khoruts, A., From stool transplants to next-generation microbiota therapeutics. Gastroenterology 2014, 146, 1573-1582.

[104] Riehle, K., Coarfa, C., Jackson, A., Ma, J. et al., The Genboree Microbiome Toolset and the analysis of $16 \mathrm{~S}$ rRNA microbial sequences. BMC Bioinformatics 2012, 13 (Suppl 1), S11.

[105] Caporaso, J. G., Kuczynski, J., Stombaugh, J., Bittinger, K. et al., QIIME allows analysis of high-throughput community sequencing data. Nat. Methods 2010, 7, 335-336.

[106] Schloss, P. D., Westcott, S. L., Ryabin, T., Hall, J. R. et al., Introducing mothur: open-source, platform-independent, community-supported software for describing and comparing microbial communities. Appl. Environ. Microbiol. 2009, 75, 7537-7541.

[107] Huson, D. H., Xie, C., A poor man's BLASTX-highthroughput metagenomic protein database search using PAUDA. Bioinformatics 2014, 30, 38-39.

[108] Li, W., Godzik, A., Cd-hit: a fast program for clustering and comparing large sets of protein or nucleotide sequences. Bioinformatics 2006, 22, 1658-1659.

[109] Haas, B. J., Gevers, D., Earl, A. M., Feldgarden, M. et al., Chimeric 16S rRNA sequence formation and detection in Sanger and 454-pyrosequenced PCR amplicons. Genome Res. 2011, 21, 494-504.

[110] Wang, Q., Garrity, G. M., Tiedje, J. M., Cole, J. R., Naive Bayesian classifier for rapid assignment of rRNA sequences into the new bacterial taxonomy. Appl. Environ. Microbiol. $2007,73,5261-5267$.
[111] Langmead, B., Salzberg, S. L., Fast gapped-read alignment with Bowtie 2. Nat. Methods 2012, 9, 357-359.

[112] Kanehisa, M., Goto, S., Sato, Y., Furumichi, M. et al., KEGG for integration and interpretation of large-scale molecular data sets. Nucleic Acids Res. 2012, 40, D109-D114.

[113] Abubucker, S., Segata, N., Goll, J., Schubert, A. M. et al., Metabolic reconstruction for metagenomic data and its application to the human microbiome. PLoS Comput. Biol. 2012, 8, e1002358.

[114] Segata, N., Izard, J., Waldron, L., Gevers, D. et al., Metagenomic biomarker discovery and explanation. Genome Biol. 2011, 12, R60.

[115] Peng, Y., Leung, H. C. M., Yiu, S. M., Chin, F. Y. L., IDBAUD: a de novo assembler for single-cell and metagenomic sequencing data with highly uneven depth. Bioinformatics 2012, 28, 1420-1428.

[116] Nielsen, H. B., Almeida, M., Juncker, A. S., Rasmussen, S. et al., Identification and assembly of genomes and genetic elements in complex metagenomic samples without using reference genomes. Nat. Biotechnol. 2014, 32, 822-828.

[117] Kurtz, S., Phillippy, A., Delcher, A. L., Smoot, M. et al., Versatile and open software for comparing large genomes. Genome Biol. 2004, 5, R12.

[118] Xu, Z., Hao, B., CVTree update: a newly designed phylogenetic study platform using composition vectors and whole genomes. Nucleic Acids Res. 2009, 37, W174-W178.

[119] Segata, N., Waldron, L., Ballarini, A., Narasimhan, V. et al., Metagenomic microbial community profiling using unique clade-specific marker genes. Nat. Methods 2012, 9, 811814.

[120] Mende, D. R., Sunagawa, S., Zeller, G., Bork, P., Accurate and universal delineation of prokaryotic species. Nat. Methods 2013, 10, 881-884.

[121] Zhang, C., Yin, A., Li, H., Wang, R. et al., Dietary Modulation of Gut Microbiota Contributes to Alleviation of Both Genetic and Simple Obesity in Children. EBioMedicine 2015, 2, 966982.

[122] Kanehisa, M., Goto, S., Sato, Y., Kawashima, M. et al., Data, information, knowledge and principle: Back to metabolism in KEGG. Nucleic Acids Res. 2014, 42, D199-D205.

[123] Caspi, R., Altman, T., Billington, R., Dreher, K. et al., The MetaCyc database of metabolic pathways and enzymes and the BioCyc collection of Pathway/Genome Databases. Nucleic Acids Res. 2014, 44, D471-D480.

[124] Overbeek, R., Olson, R., Pusch, G. D., Olsen, G. J. et al., The SEED and the Rapid Annotation of microbial genomes using Subsystems Technology (RAST). Nucleic Acids Res. 2014, 42, D206-D214.

[125] Saier, M. H., Reddy, V. S., Tsu, B. V., Ahmed, M. S. et al., The Transporter Classification Database (TCDB): recent advances. Nucleic Acids Res. 2016, 44, D372-D379.

[126] Burgard, A. P., Pharkya, P., Maranas, C. D., Optknock: a bilevel programming framework for identifying gene knockout strategies for microbial strain optimization. Biotechnol. Bioeng. 2003, 84, 647-657. 
[127] Schellenberger, J., Que, R., Fleming, R. M. T., Thiele, I. et al., Quantitative prediction of cellular metabolism with constraint-based models: the COBRA Toolbox v2.0. Nat. Protoc. 2011, 6, 1290-1307.

[128] Greenblum, S., Turnbaugh, P. J., Borenstein, E., Metagenomic systems biology of the human gut microbiome reveals topological shifts associated with obesity and inflammatory bowel disease. Proc. Natl. Acad. Sci. U S A 2012, 109, 594-599.

[129] Xu, N., Liu, J., Ai, L., Liu, L., Reconstruction and analysis of the genome-scale metabolic model of Lactobacillus casei LC2W. Gene 2015, 554, 140-147.

[130] El-Semman, I. E., Karlsson, F. H., Shoaie, S., Nookaew, I. et al., Genome-scale metabolic reconstructions of Bifidobacterium adolescentis L2-32 and Faecalibacterium prausnitzii A2-165 and their interaction. BMC Syst. Biol. $2014,8,41$.

[131] Ji, B., Nielsen, J., From next-generation sequencing to systematic modeling of the gut microbiome. Front. Genet. 2015, 6, 219.

[132] Moriya, Y., Shigemizu, D., Hattori, M., Tokimatsu, T. et al.,
PathPred: an enzyme-catalyzed metabolic pathway prediction server. Nucleic Acids Res. 2010, 38, W138-W143.

[133] Ibrahim, M., Anishetty, S., A meta-metabolome network of carbohydrate metabolism: interactions between gut microbiota and host. Biochem. Biophys. Res. Commun. 2012, 428, 278-284.

[134] Steinway, S. N., Biggs, M. B., Loughran, T. P., Papin, J. A. et al., Inference of network dynamics and metabolic interactions in the gut microbiome. PLoS Comput. Biol. 2015, $11, \mathrm{e} 1004338$.

[135] Ni, Y., Li, J., Panagiotou, G., A molecular-level landscape of diet-gut microbiome interactions: toward dietary interventions targeting bacterial genes. MBio 2015, 6, e01263e01215.

[136] Saito, R., Smoot, M. E., Ono, K., Ruscheinski, J., A travel guide to Cytoscape plugins. Nat. Methods 2012, 9, 10691076.

[137] Kristensen, N. B., Bryrup, T., Allin, K. H., Nielsen, T. et al., Alterations in fecal microbiota composition by probiotic supplementation in healthy adults: a systematic review of randomized controlled trials. Genome Med. 2016, 8, 52. 